\title{
SPATIAL REPARTITION OF CURRENT FLUCTUATIONS IN A SCANNING TUNNELING MICROSCOPE
}

\author{
Jerome Lagoute, Tomaso Zambelli, Stephane Martin and Sebastien Gauthier \\ Centre d'Elaboration de Matériaux et d'Etudes Structurales 29, rue Jeanne Marvig 31055 Toulouse, France \\ e-mail: lagoute@cemes.fr, zambelli@cemes.fr, martin@lget.ups-tlse, gauthier@cemes.fr \\ (Accepted August 24, 2001)
}

\begin{abstract}
Scanning Tunneling Microscopy (STM) is a technique where the surface topography of a conducting sample is probed by a scanning metallic tip. The tip-to-surface distance is controlled by monitoring the electronic tunneling current between the two metals. The aim of this work is to extend the temporal range of this instrument by characterising the time fluctuations of this current on different surfaces. The current noise power spectral density is dominated by a characteristic $1 / \mathrm{f}$ component, the physical origin of which is not yet clearly identified, despite a number of investigations. A new I-V preamplifier was developed in order to characterise these fluctuations of the tunnelling current and to obtain images of their spatial repartition. It is observed that their intensity is correlated with some topographical features. This information can be used to get insights on the physical phenomena involved that are not accessible by the usual STM set-up, which is limited to low frequencies.
\end{abstract}

Keywords: 1/f noise, microscope, noise, STM, tunnel-junction.

\section{INTRODUCTION}

STM images are affected by noise that can be seen as a limitation of their quality. Hence, different authors gave techniques to restore noisy images (Stoll et al., 1987; Abraham et al., 1988). Another point of view is to use current fluctuations for obtaining information on the studied surface. The STM device includes a tip, that scans the surface, supported by a piezoelectric positioning system. The resonance frequency of this device limits the scanning speed. In the best conditions, an image is obtained in the order of 1 second restricting the accessible time resolution of physical processes occurring on the surface. Dynamics studies (Binnig et al., 1986; Koslowski et al., 1993) as well as new device development (Koslowski et al., 1993; Möller et al., 1993), using noise studies, justify an increasing interest for the investigation of noise. A spectral analysis of the tunnelling current leads to the observation of a $1 / \mathrm{f}$ low-frequency contribution, the origin of which is still unknown in spite of many propositions (Dutta and Horn, 1981). Its understanding could lead to a new way of surface investigation by means of STM images of the spatial variation of $1 / \mathrm{f}$ fluctuations (Sugita $e t$ al., 1996). In the following, we present simultaneously obtained images of the spatial repartition of the fluctuations and the topography on different surfaces.

\section{MATERIALS AND METHODS}

We use a home-made STM operating in ultrahigh vacuum $\left(\mathrm{P}<10^{-8} \mathrm{~Pa}\right)$. Electrochemically etched tungsten tips have been prepared by heating and ion sputtering. The copper surfaces were cleaned by repeated cycles of sputtering $\left(6.10^{-3} \mathrm{~Pa}\right.$ Ar pressure $)$ and annealing (700K).

We developed for this study a new I-V converter with a gain of $10^{8} \mathrm{~V} / \mathrm{A}$ and a bandwidth of $370 \mathrm{kHz}$. The time recording of the current is made by a computer using an A/D converter board with an antialiasing filter (100 kHz cutoff frequency).

During the scanning, the tip height is regulated by an analogical feedback loop. To study the spatial variation of the current, a lock-in amplifier was directly connected to the output of the I-V converter, in parallel with the feedback loop (Fig. 1). The output of the lockin amplifier gives the contribution of the current fluctuations around a chosen frequency in a window of $500 \mathrm{~Hz}$. This signal is recorded simultaneously with the topography. Hence, our device gives at the same time the tip height map (topography) and the current fluctuation map. The physical variable of interest is the power spectral density of the current fluctuations averaged in the $500 \mathrm{~Hz}$ window, which is given by the variance of the previous image, obtained by a simple numerical calculation. 


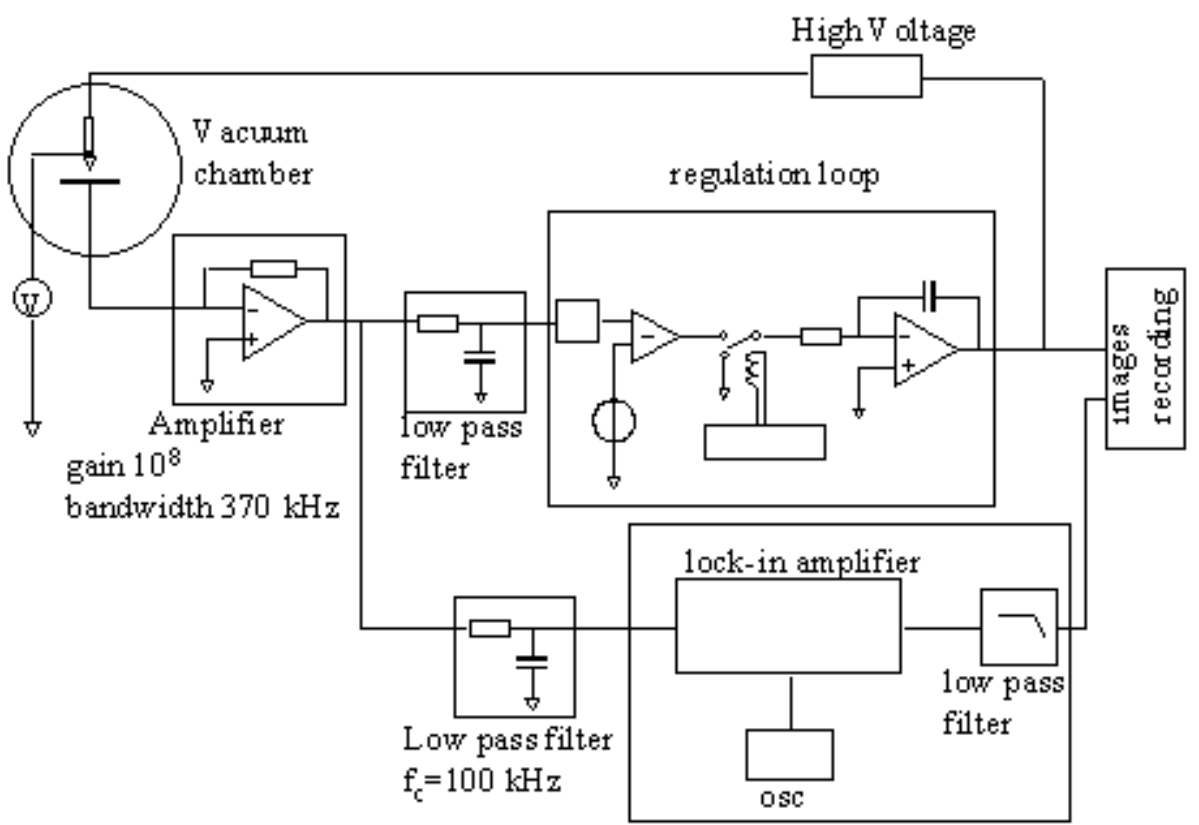

Fig. 1. Experimental device for recording topographic and fluctuations images.

The output of the amplifier is connected to a feedback loop that controls the tip height. For that, a high voltage is applied to the piezoelectric tube that supports the tip. At the same time, the lock-in amplifier filters the current fluctuations, and the resulting signal is recorded.

\section{RESULTS}

The tunnel current noise power spectral density is typically (Fig. 2) a combination of $1 / \mathrm{f}$ and white noise (thermal and shot-noise). The thermal noise is due to the temperature and the resistance of the junction
$\left(\mathrm{S}_{\text {thermal }}=4 \mathrm{kT} / \mathrm{R}\right)$. The shot noise is due to the quantification of the electric charge and is proportional to the current $\left(\mathrm{S}_{\mathrm{shot}}=2 \mathrm{eI}\right)$. The $1 / \mathrm{f}$ current fluctuations dominate the spectrum at low frequencies. The corresponding power spectrum obeys the empirical Hooge's formula (Hooge, 1969):

$$
S_{1 / \mathrm{f}}=\frac{\alpha \mathrm{I}^{2}}{\mathrm{f}^{\beta}},
$$

with a $\beta$ value between 0.8 and 1.4. The current is maintained at a constant value, so the variation of noise results from fluctuations of $\alpha$ and $\beta$ parameters.

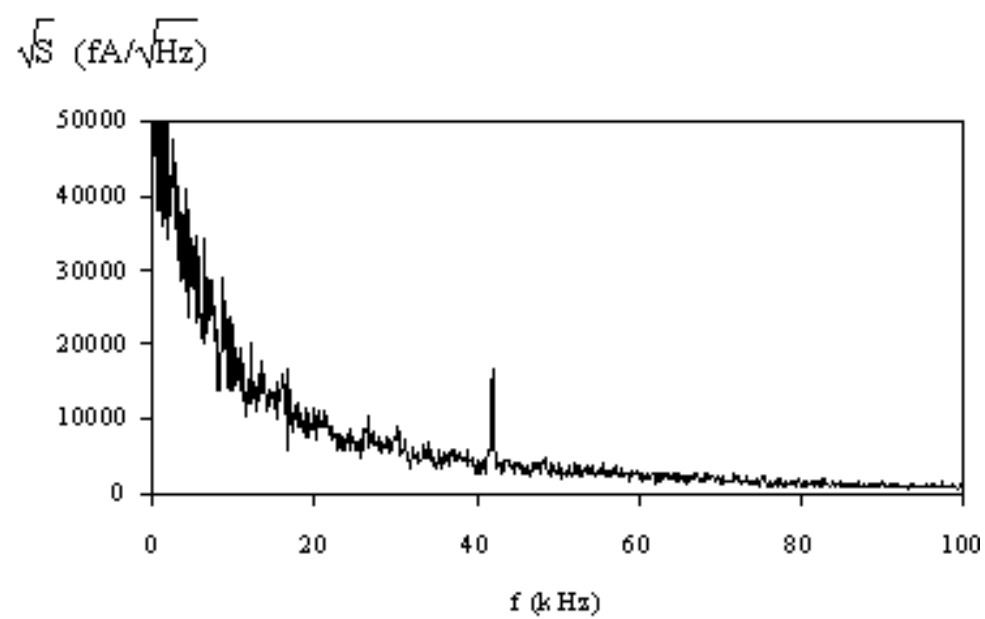

Fig. 2. A typical power spectrum of the tunnel current. The $1 / f$ noise dominates the spectrum at low frequencies. The peak at $42 \mathrm{kHz}$ is an artefact. 
In a first series of experiments, the beginning stages of the oxidation of a $\mathrm{Cu}(100)$ surface were investigated. The $\mathrm{O}_{2} / \mathrm{Cu}(100)$ system has been the subject of numerous investigations (Jensen, 1990). It is known that the surface reconstructs in a $(2 \sqrt{2} \times \sqrt{ } 2) \mathrm{R} 45^{\circ}$ structure, which forms by expelling one quarter of the copper atoms from the surface layer. These ejected atoms renucleate, forming characteristic linear islands, oriented in the <001> crystallographic directions. Fig. 3 shows the topography of the surface after an exposure to $2.66 \times 10^{-2} \mathrm{~Pa}^{-1}{ }^{-1}$. It shows three types of spatial features: terraces, step edges and the expected linear islands. The $0.18 \mathrm{~nm}$ step edges, separating two terraces, were present on the surface before the $\mathrm{O}_{2}$ exposure. On the other hand, the linear islands appear during the oxidation process. The fluctuation map simultaneously recorded with the topography presents a uniform level on the terraces. The step edges do not appear distinctly in the image $3 \mathrm{~b}$. On the contrary, the linear islands are characterized by a clear increase in the fluctuation intensity.

A second series of experiments were conducted with bis-porphyrin molecules (Fig. 4) deposited on a $\mathrm{Cu}(111)$ surface by sublimation from a heated filament positioned in front of the sample. This method is likely to produce molecular dissociation. At the time of this writing, the complete analysis of the images has not been done so it is not possible to give the precise structure of the adsorbate. Nevertheless, the molecular lattice period of $1.5 \mathrm{~nm}$ observed on Fig. 6a is compatible with single porphyrin molecules (Jung, 1997).

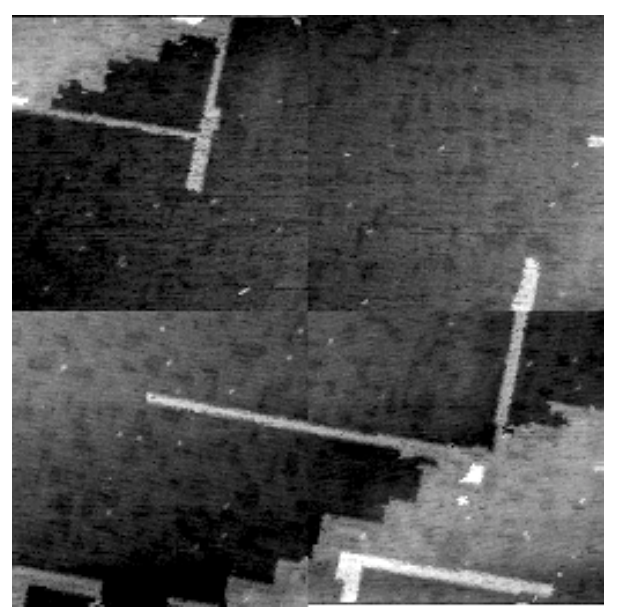

a)

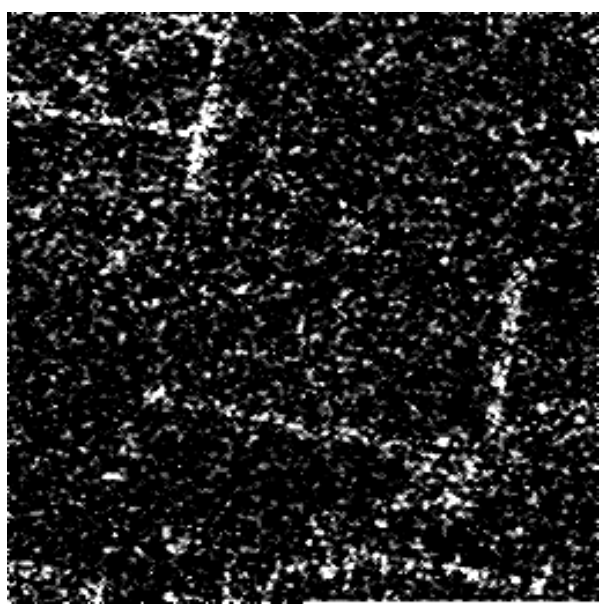

b)

Fig. 3. a) topography of a partly oxidised $\mathrm{Cu}(100)$ surface. b) spatial variation of the current noise power spectral density in the range of $9.75-10.25 \mathrm{kHz}$. The size of the images is $29 \times 29 \mathrm{~nm}^{2}$.

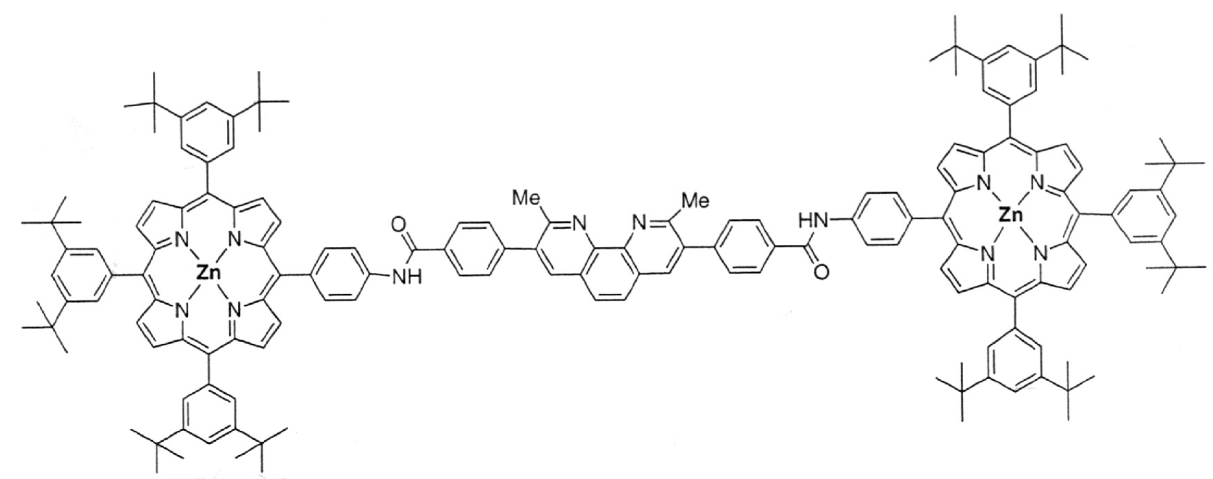

Fig. 4. Chemical structure of the bis-porphyrin molecules. 
The topography (Fig. 5a) shows noisy terraces and areas on which molecules are arranged in a regular lattice. The current fluctuations map (Fig. 5b) shows a clear correlation with the topography. The step edge does not appear in the fluctuation map and the molecular lattice is characterised by a decrease in the fluctuations intensity with respect to the level observed on the terraces.

A contrast can also be distinguished at the molecular scale (Fig. 6). Furthermore, the noise on top of a molecule is lower than between the molecules.

\section{INTERPRETATION}

The interpretation of these data is not straightforward because of the different effects that could contribute. First, experimental artefacts have to be excluded. In particular, one has to choose a working frequency well outside the range where instabilities of the feedback loop regulating the tunnelling current can occur. It has been checked that even when crossing features like step edges, the noise generated by the control system is negligible at the frequencies chosen for the fluctuation images. This can be verified on Fig. 5, where the step observed on the topography does not contribute to the noise map, despite a relatively large scanning speed of the tip. This observation permits also to reject the possibility that the noise would be produced by mechanical oscillations of the tip in the horizontal plane.

An important class of dynamical processes occurring on surfaces is surface diffusion. Indeed, fluctuations of the tunnelling current observed by Binnig et al. (1986) on the $\mathrm{O}_{2} / \mathrm{Ni}(100)$ system were interpreted by surface diffusion of oxygen atoms crossing the tip-sample region.

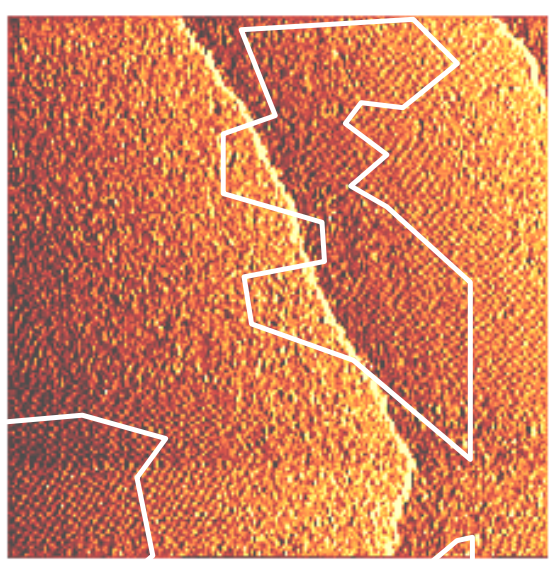

a)

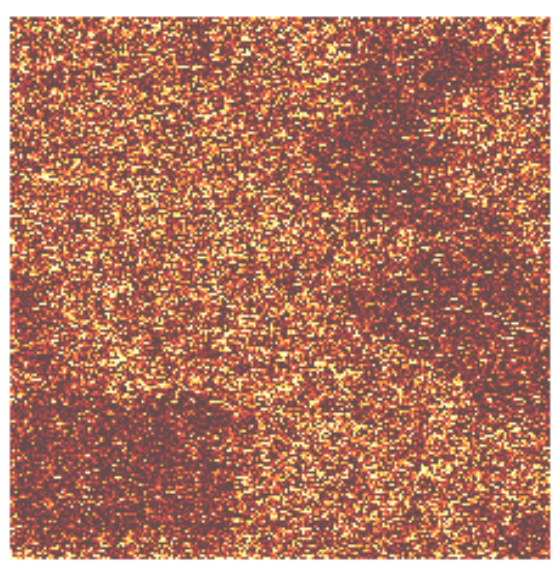

b)

Fig. 5. a) Topography of a $\mathrm{Cu}(111)$ surface resulting from the deposition of bis-porphyrin molecules. $b$ ) Areas where the molecules are observed appear as dark patches in the fluctuation map. These images were obtained in the same conditions as Fig. 3. Image size $72 \times 72 \mathrm{~nm}^{2}$.

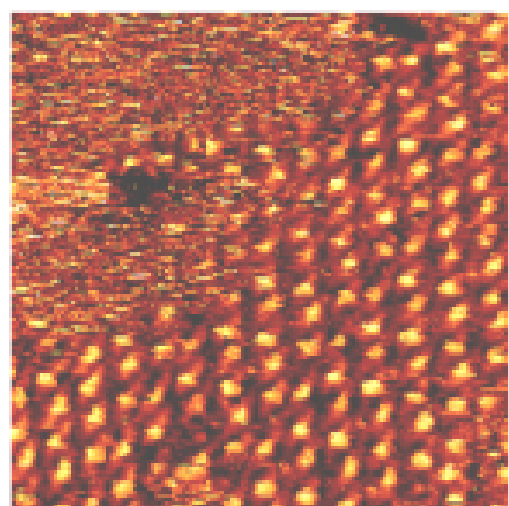

a)

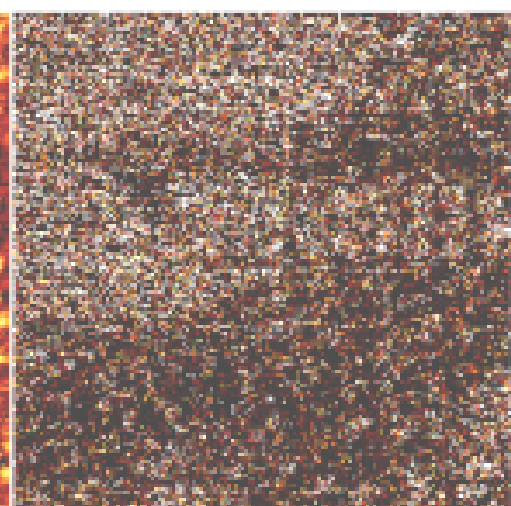

b)

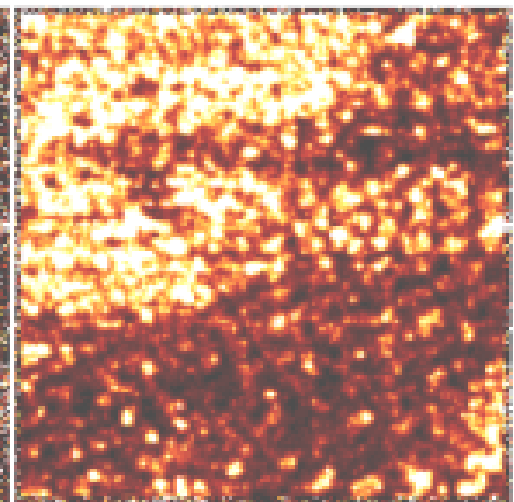

c)

Fig. 6. a) Topography of a Cu(111) surface resulting from the deposition of bis-porphyrin molecules. b) The molecules are resolved in the fluctuation map (raw data) and c) (filtered fluctuation map). These images were obtained in the same conditions as Fig. 3. Image size: $18 \times 18 \mathrm{~nm}^{2}$. 
Theoretical evaluation of this phenomenon has been made following this pioneering work (Sumetski et al., 1994). It seems clear that the fluctuations observed on $\mathrm{O}_{2} / \mathrm{Cu}(100)$ should be interpreted in the same terms, with the complication that the substrate is oxidised during the process. Dioxygen molecules, oxygen atoms and copper atoms are diffusing on the substrate. Furthermore, the sample becomes heterogeneous as oxidation proceeds. Nevertheless, a clue is given by the elongated shape of the islands: it implies that they grow by matter incorporation at their extremity tip where reactive sites have to be located. Diffusing species are collected by the steps limiting the long sides of the islands and diffuse in this 1-dimensional channel before being incorporated at the extremity of the island. Due to this 1D character, the probability that a diffusing species crosses the tip-surface junction is higher when the tip is above these steps than above a terrace site where the diffusion is 2-dimensional. In this explication, the steps limiting the elongated islands play the role of a bottleneck for the diffusing species.

In the second series of experiments, the lower fluctuation level observed on the molecular lattice could result from a limitation in the thermal displacement of the molecules. Because of thermal agitation, the molecules tend to randomly diffuse on the surface. On a terrace where the adsorbates can be considered as free in two dimensions a molecule can cross the tip-sample region in its whole length. A current fluctuation corresponding to the total height of the molecule (as measured by STM) is then recorded. But in the adsorbate layer, intermolecular interactions limit molecular lateral displacements to distances largely smaller than the size of a molecule. The amplitude of the resulting current fluctuation is then smaller in the molecular island than in the terrace because it is related to a fraction only of the total height of the molecule. Furthermore, the contrast at the molecular scale can be assumed to be due to the shape of the molecule. On the top, the profile is flat, and a lateral displacement cause little modification of the tip-to-molecule distance. Between two molecules, the tip is sensitive to the current tunneling through lateral faces of the molecules, that are relatively abrupt. When the molecules move, the current changes more. This assumption is consistent with the observation made on our images showing a higher fluctuation level between the molecules than on top of them.

\section{CONCLUSION}

This study should be considered as preliminary.
Much work remains to be done to reach a good understanding of the data. Nevertheless, these results show that this approach is promising to extend the dynamical range of STM, which is fairly limited in its conventional utilisations.

\section{ACKNOWLEDGEMENTS}

The bis-porphyrin molecules were kindly supplied by J. C. Chambron (Laboratoire de Chimie OrganoMinérale Université Louis Pasteur, Institut Le Bel. 4, rue Blaise Pascal F-67000 Strasbourg France). This work was supported by the European Community under the "Information Society Technologies" program NANOMOL.

\section{REFERENCES}

Abraham DW, Williams CC, Wickramasinghe HK (1988). Noise reduction technique for scanning tunneling microscopy. Appl Phys Lett 53:1503-5.

Binnig G, Fuchs H, Stoll E (1986). Surface diffusion of oxygen atoms individually observed by STM. Surf Sci 169:L295-L300.

Dutta P, Horn PM (1981). Low frequency fluctuations in solids: 1/f noise. Rev Mod Phys 53(3):497-516.

Hooge FN (1969). 1/f noise is no surface effect. Phys Lett 29A(3):139-40.

Jensen F, Besenbacher F, Laegsgaard E, Stensgaard I (1990). Dynamics of oxygen-induced reconstruction of $\mathrm{Cu}(100)$ studied by scanning tunneling microscopy. Phys Rev B42:9206-9.

Jung TA, Schlittler RR, Gimzewski JK, Tang H, Joachim C (1997). Conformational identification of individual adsorbed molecules with the STM. Nature 386:696-8.

Koslowski B, Baur C, Möller R, Dransfeld K (1993). Atomic scale variation of current noise on GaAs (110) detected by a scanning tunneling microscope. Surf Sci 280:106-14.

Möller R, Esslinger A, Koslowski B (1990). Thermal noise in vacuum scanning tunneling microscopy at zero bias voltage. J Vac Sci Technol A 8(1):590-3.

Stoll E, Marti O (1987). Restoration of scanning-TunnelingMicroscope data blurred by limited resolution, and hampered by 1/f-like noise. Surf Sci 181:222.

Sugita S, Mera Y, Maeda K (1996). Origin of low frequency noise and 1/f fluctuations of tunneling current in scanning tunneling microscopes. J Appl Phys 79(8):4166-73.

Sumetski M, Kornyshev AA, Stimming U (1994). Adatom diffusion characteristics from STM noise: theory. Surf Sci 307-309:23-7. 